\section{A giant cholesteatoma of the mastoid extending into the foramen magnum: A case report and review of literature}

\author{
Seidu A. Richard, ${ }^{1-3}$ Li Qiang,1 \\ Zhi Gang Lan, ${ }^{1}$ Yuekang Zhang, ${ }^{1}$ \\ Chao You' 1 \\ 1Department of Neurosurgery, West \\ China Hospital, Sichuan University, \\ China; 2Department of Immunology, \\ Jiangsu University, China; ${ }^{3}$ Department \\ of Surgery, Volta Regional Hospital, Ho, \\ Ghana
}

\begin{abstract}
Cholesteatomas are very rare benign, progressive lesions that have embryologic derivation and usually result in progressive exfoliation and confinement of squamous epithelium behind an intact or preciously infected tympanic membrane. To the best of our understanding no reports demonstrates the extension of cholesteatoma from the
\end{abstract} temporal bone into the foramen magnum. We therefore present a case of cholesteatoma extending down into the foramen magnum. We report a case of 67year-old man with a giant cholesteatoma extending into the foramen magnum without substantial destruction of the mastoid and petrous temporal bones. The patient's major symptoms were recurrent tinnitus in the left ear and dizziness with unilateral conductive hearing loss. A working diagnosis of cholesteatomas was made combining the symptoms and magnetic resonance imaging findings. He was then successfully operated on with very minimal postoperative complications. Cholesteatomas originating from the mastoid bone often linger with the patients for many years in a subclinical state and progress into a massive size before causing symptoms. Patients with unilateral conductive hearing loss who are otherwise asymptomatic and have a normal tympanic membrane should be suspected with a progressive cholesteatoma. Cholesteatoma should be one of the working diagnosis when an elderly patient present with unilateral conductive hearing loss that is associated with tinnitus and dizziness.

\section{Introduction}

Cholesteatomas are benign, slowly progressive lesions that are usually found in the temporal bone.1-5 They are epidermoid cysts of embryologic origin that result in progressive desquamation and trapping of squamous epithelium behind an intact tympanic membrane. ${ }^{6}$ They can be congenital or acquired.1,4,5 They can be classified into supralabyrinthine, infralabyrinthine, massive labyrinthine, infralabyrinthine-apical, and apical.1,4 They have the penchant for the petrous apex, skull base, and internal auditory canal. Also, these lesions may infiltrate other vital soft tissue structures such as the sigmoid sinus, jugular vein and artery, and the cerebellopontine angle. $1,4,5$ Symptoms associated with cholesteatomas includes vertigo, tinnitus, otorrhea, and otalgia.1,7 To the best of our understanding no data has reported cholesteatoma that extends from the temporal bone into the foramen magnum. We therefore present a case of cholesteatoma extending down into the foramen magnum and review of literature on the pathogenesis of cholesteatoma.

\section{Case Report}

We present a case of 67-year-old man with recurrent tinnitus in the left ear and dizziness for over one-year duration. He had mild unilateral hearing loss, no otorrhea, vertigo, pain, cognitive impairment, vomiting, facial numbness, hypoesthesia, blurred vision, choking and movement disorders. Past history of ear infection was unremarkable. All other systems were unremarkable. There was no family history of such disease. He did not have any ear surgeries prior to the presentation. On examination, there were no obvious deformities around the ears. Otoscopy revealed an intact tympanic membrane with no visible pathology in the mesotympanum. Examination of cranial nerves was unremarkable. Audiometry demonstrated total hearing loss with reduced middle ear compliance in the left ear. All laboratory investigations as well as other ancillary investigations (CXR, ECG etc) were normal.

Pre-operative MRI done showed a lesion that is hypo-intense on T1, hyperintense on $\mathrm{T} 2$ and hypointensive on FLAIR and showed no enhancement with gadolinium administration. The lesion was about $3.0 \times 2.5 \times 2.5 \mathrm{~cm}$ in diameter. The lesion displaced the medulla oblongata to the right side and compressed the bilateral cerebellum hemispheres. The third, fourth and bilateral ventricles are slightly enlarged
Correspondence: Yuekang Zhang, Department of Neurosurgery, Post Graduate Training Centre, West China Hospital, Sichuan University; 37 Guo Xue Xiang Road, Chengdu, Sichuan 610041, P.R. China. Tel.: +86.18980601975 - Fax: +86.02885422490. E-mail: neurovip@yeah.net

Key words: Cholesteatoma, Case report, Dizziness, Epidermoid Cyst, Hearing loss, Tinnitus.

Contributions: SAR conceived the project and designed the study; SAR, LQ and ZQL collected patient's data; YZ and CY provided technical assistance in the study; SAR prepared the illustration, analyzed data and wrote the paper.

Conflict of interest: the authors declare no potential conflict of interest.

Funding: none.

Received for publication: 31 January 2018. Accepted for publication: 4 February 2018.

This work is licensed under a Creative Commons Attribution NonCommercial 4.0 License (CC BY-NC 4.0).

(C) Copyright S.A. Richard et al., 2018 Licensee PAGEPress, Italy

Neurology International 2018; 10:7625

doi:10.4081/ni.2018.7625

with mild obstructive hydrocephalus. Left frontal white matter showed a spotty lesion that is hypo-intense on $\mathrm{T} 1$ and Hyperintense on $\mathrm{T} 2$ which could be the beginning of mild degenerative process. There were no abnormalities on the temporal bone and for that matter the whole skull. A working diagnosis epidermoid or arachnoid cyst was made combining the symptoms and MRI findings (Figure 1).

After general anesthesia, the patient was put in the park-bench position. Routing use of inferior cranial nerves monitoring such as electromyographic (EMG) and auditory brainstem responses (ABRs) was carried out to maximize the preservation of the cranial nerves. We used the suboccipital paramedian approach. A linear suboccipital paramedian incision was made and extended down to the occipital hairline. A highspeed miller was used to perform a suboccipital paramedian craniotomy. Additionally, posterior rim of foramen magnum and the lamina of $\mathrm{C} 1$ were also removed to gain wider exposure of the lesion. After placing several tack-up stitches to the dura, the lesion was observed lying between jugular foramen and the foramen 
magnum. The lesion was about $3.0 \times 2.5 \times 2.5$ $\mathrm{cm}$ in diameter. The microscope was used throughout the operation. The lesion was completely suctioned out without any cranial nerves dysfunction. Post-operative CTscan done confirmed total resection of the lesions with very slight edema around the brain stem (Figure 2). Samples of the lesion are as shown in (Figure 3). Pathology report confirmed cholesteatoma or epidermoid cyst (Figure 4). The patient had a good recovery and discharge home on the seventh day after operation. Hearing improved markedly. A scheduled review was arranged on the third month after operation. One and half year follow-up show no recurrence of the lesion.

\section{Discussion}

Cholesteatomas or epidermoid cysts originate from progressive desquamation of the epithelium.1,2,4,5,7 They are classified into congenital or acquired base on their pathogenesis.1,4,5 In our case, it was very difficult to arrive at a definitive conclusion on whether the lesion is congenital or acquired but we believe it has a congenital origin. They can further be categorized into supralabyrinthine, infralabyrinthine, massive labyrinthine, infralabyrinthine-apical, and apical base on their location. 1,4 Furthermore, four potential directions of extension outside the borders of the temporal bone was described by Wayoff et al. as follows: i) subcutaneous extension toward the external mastoid cortex or temporal squama; ii) suprapetrous extension toward the internal auditory canal; iii) forward extension toward the geniculate ganglion; iv) downward and forward extension into the hypotympanic region to involve the internal jugular vein and the carotid artery. 8,9 The most frequent presenting symptoms are otalgia, malodorous otorrhea, and hearing loss. 10-12 Cholesteatomas have the possibility of causing aggressive complications such as brain abscess and meningitis in the central nervous making the lesions hypothetically lethal. Chronic polymicrobial infection and bony erosion usually leads to these secondary complications. ${ }^{13}$

Most cholesteatomas reported in literature are usually congenital. Regardless of their congenital origin, they may start to give symptoms at practically any age and may remain asymptomatic for long periods of the life of the patients although majority of patients' symptoms first appear at an elderly age. ${ }^{13}$ Congenital cholesteatoma, by definition originate from areas of keratinizing epithelium within the middle ear cleft. Numerous theories arose in connection to pathogenesis which includes: i) the existence of an ectopic epidermis rest, ii) ingrowth of meatal epidermis, iii) metaplasia following infection/inflammation, and fascinatingly, iv) reflux of amniotic fluid containing squamous epithelium in utero into the middle ear. Nonetheless, the epithelial rest theory is generally accepted. ${ }^{13}$ Congenital cholesteatomas in the middle ear frequently present with cunning hearing loss during childhood years and are usually found incidentally behind the tympanic membrane during routine otoscopy.2,14 Nevertheless, congenital cholesteatomas may be absolutely asymptomatic, particularly when their origin is from the mastoid where the diagnosis occurs only as an incidental finding on imaging hence the diagnosis is often belated until mid to late adulthood because of the asymptomatic nature of the disease.2,14 In such cases, the most common symptoms are dizziness, pain, or swelling that may be localized to the temporal area of the head or to the neck. ${ }^{2,14}$

Even though the pathophysiology of the
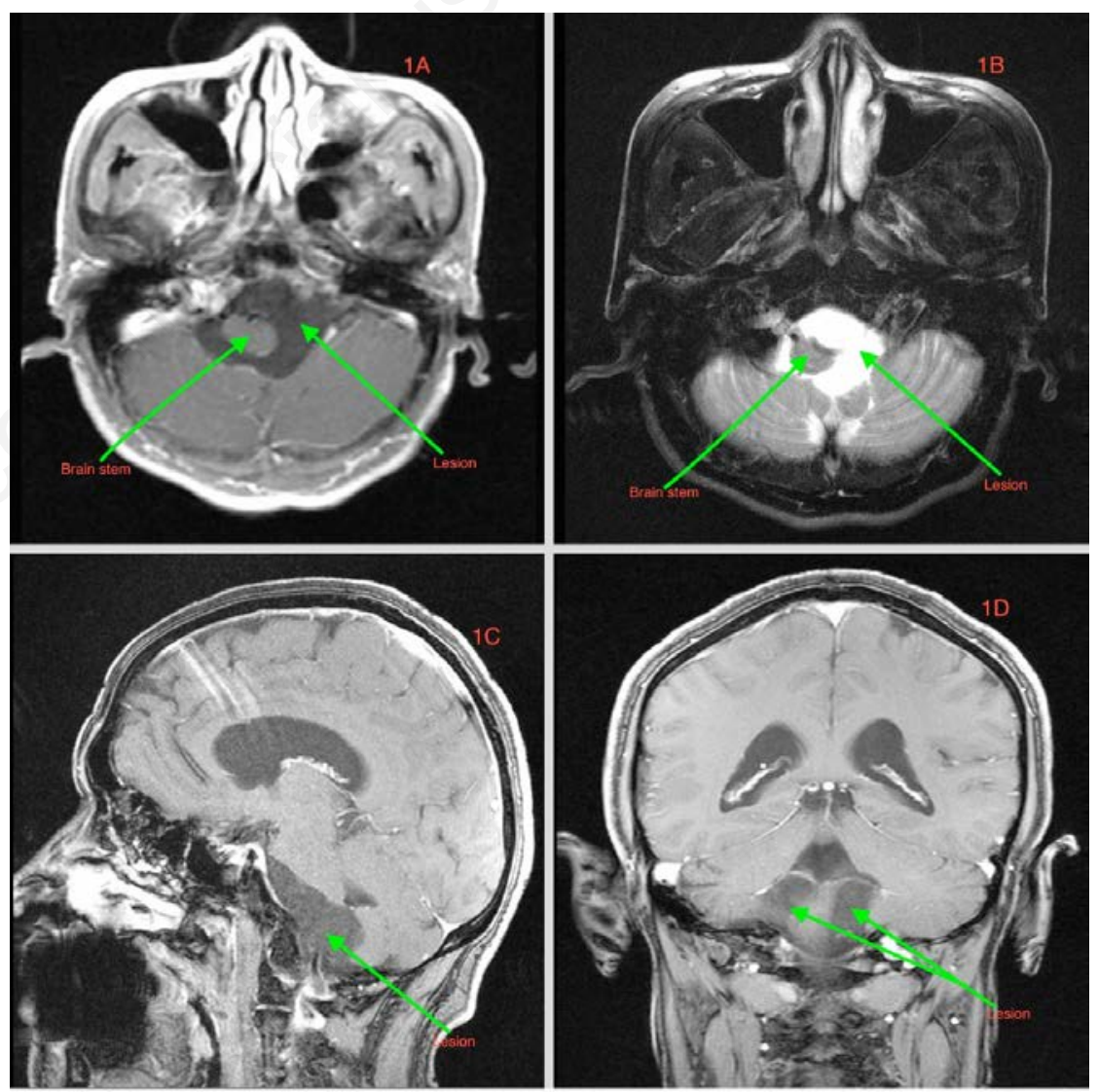

Figure 1. A,B) are axial T1 and T2 respectively. The lesion is hypo-intense on T1, hyperintense on T2. The lesion compressed the medulla oblongata on the right side and the hemisphere bilaterally with intense pressure on the medulla oblongata and the cerebellum. C) is a sagittal view of the lesion on T1. The lesion is lying on the posterior fossa floor and extending to the foramen magnum. D) is coronal View of the lesion on T1; showing the third, fourth and bilateral ventricles which are slightly enlarged with mild obstructive hydrocephalus. The lesion is hypo-intense.

acquired cholesteatoma still remains to be distinctly clarified, it is believed to be multifactorial, as many theories have been recommended and researched on. ${ }^{13}$ It has been demonstrated that iatrogenic or non-iatrogenic tympanic membrane trauma such as invaginon, displacement, retraction or tympanic cavity mucosa disease, ear infection, and Eustachian tube dysfunction are likely to initiate acquired cholesteatoma pathogenesis. ${ }^{13,15}$ The pathogenesis of acquired aural cholesteatoma has also been explained by four basic theories: i) invagition pocket hyperplasia, iii) epithelial in-growth through perforation (the migration theory), and iv) squamous metaplasia of middle ear epithelium. ${ }^{13}$ The most widely accepted pathogenesis of acquired cholesteatoma is deepening retraction pocket that, when obstructed, desquamated keratin cannot be cleared from the recess, and cholesteatoma

[Neurology International 2018; 10:7625]

[page 29] 
results. ${ }^{13}$ The source of the retracted pocket cholesteatomas is believed to be the dysfunction of the Eustachian tube or otitis media with effusion with resultant negative middle ear pressure (ex vacuo theory). Normally, the pars flaccida, being less fibrous and less resistant to displacement, is the source of the cholesteatoma. ${ }^{16}$ Chronic inflammation appears to play an essential part in multiple etiopathogenic mechanisms of acquired cholesteatoma.

The use of common imaging modalities such as high-resolution CT scan and MRI are important to detect and also define the extent of the lesion, which is fundamental for planning surgical intervention.2,3,14,17 CT scans usually confirm the existence and offer a suggestion on the size and magnitude of the lesion, and MRI differentiates cholesteatomas from other soft tissue masses like neuromas, schwannomas, or metastases while DWI also further differentiate cholesteatomas from other cystic masses. ${ }^{2,3,14}$ Typical sequences used in the diagnosis of cholesteatoma are T1-weighted, T2-weighted sequences. Cholesteatoma have a signal close to the cerebellum, that is

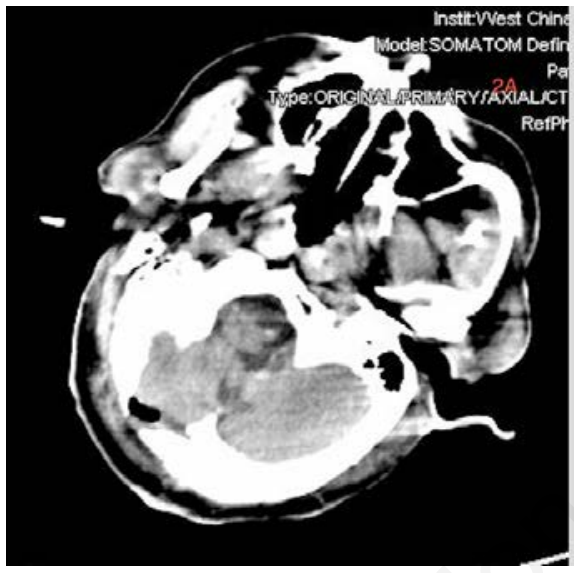

Figure 2. Postoperative CT-Scan showing total resection of the lesions with very mild edema.

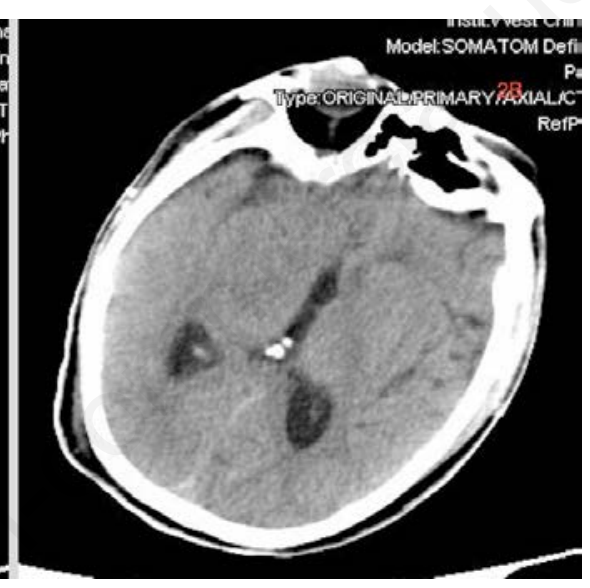

hypo-intense signal on T1, moderate hyperintense signal on $\mathrm{T} 2$, round or oval form, can be edging by a hyper-intense signal in T2 phase. ${ }^{18}$ Recently, the use of more newer sequence type diffusion that is echo-planar images (EPI-DWI) in which cholesteatoma is seen as a hyper-intense signal due to the restriction in water diffusion and perhaps oily consistency at the cystic part because of keratin. ${ }^{15,18-21}$ DW-non-EPI is a longer sequence which has effectively replaced $\mathrm{T} 1$ sequence post-contrast delayed. It also helps to tackle avoidable second-look operation at 6-18 months after surgery. 5,18,22,23

The only effective treatment for cholesteatoma is surgery. ${ }^{24}$ Currently, there are no medical treatments that has been demonstrated effective for the treatment of this disease, except the treatment of the secondary infectious processes in the cases of acquire cholesteatoma. ${ }^{13}$ We propose that microscopic suboccipital paramedian approach is highly recommended, because it allows for total or subtotal tumor removal under a clear field of vision, and is relatively simpler and easier. The microscopic approach also enables the surgeon visualize

\section{d}

-

vital cranial nerves in the posterior fossa and hence avoid injuring them during the operation. ${ }^{11}$ Weidyasekara et al. are of the view that a combined transmastoid and middle fossa approach can be used to resect huge acquired cholesteatoma that protrudes into the middle fossa. ${ }^{25}$ However, Burggraaff et al. are of the view that not all patients require combine surgery. They explain that majority of patients only need one operation to remove the lesion. ${ }^{26}$ Surgical approach to the lesions is always determined by the location of the lesion, the anatomical orientation of the internal carotid canal and the jugular bulb, as well as the state of hearing, whether intact or lost.1,27 The purpose of the surgical approach in cases of temporal bone involvement is to provide clear views of the middle and posterior fossa, dura, carotid artery, lateral sinus and jugular bulb as well as facial nerve notwithstanding the complexity of the anatomy. 1,5 In our case the lamina of $\mathrm{C} 1$ was removed to enable us gain clearer view of the lesion that was bulging through the foramen magnum. Facial nerve function must always be assessed before ear and lateral skull base surgery because preoperative dysfunction has been linked to higher risk of postoperative facial nerve palsy especially in cranial nerve schwannoma surgeries. ${ }^{28-30}$ However, most patients who had preoperative normal facial nerve function (House-Brackman I) preserved function postoperatively. ${ }^{25,26,31}$ Facial nerve function is most frequently perceived by means of electromyographic (EMG) monitoring during surgery. Also, auditory nerve is perceived by recording the auditory brainstem responses (ABRs) during surgery at the internal auditory canal (IAC) or cerebellopontine angle (CPA).28,29 Almost all the patients operated on had good post-operative recovery as reported in literature.

Even though cholesteatoma is hyperproliferative, it does not demonstrate typical

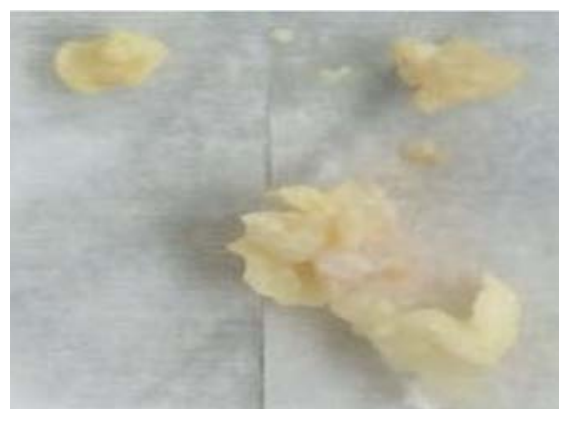

Figure 3. Samples of the lesion after operation.

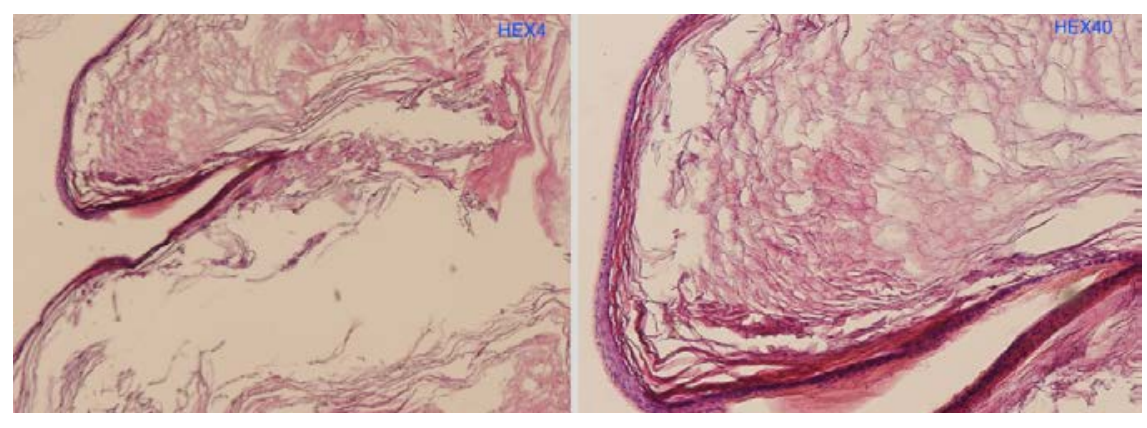

Figure 4. Hematoxylin and Eosin $4 \times$ and $40 \times$ images of the lesion. 
characters of neoplasia since it does not metastasize or genetically unstable. ${ }^{13}$ The initiation of cholesteatoma formation appears to be associated to both internal molecular dysregulation and external stimuli in the form of pro-inflammatory cytokines, growth factors and/or bacterial toxins. There is usually a disproportion and a vicious circle of epithelial proliferation, keratinocyte differentiation and maturation, prolonged apoptosis, and disturbance of self-cleaning mechanisms. ${ }^{13}$ It is known that bacteria inside the retraction pocket produce some antigens, which will activate different cytokines and lytic enzymes, these cytokines lead to the activation and maturation of osteoclasts with the consequence of degradation of extracellular bone matrix and hyperproliferation, bone erosion and ultimately development of the disease. 13,32

\section{Conclusions}

Our case is very special in the sense that it is a very rare occurrence. To the best of our knowledge no literature has review a case of massive cholesteatoma extending into foremen magnum although some literature has described massive cholesteatoma involving the temporal and petrous bones with mastoid origin. The pathogenesis of Cholesteatomas whether congenital or acquired may grow silently over many years and develop into a massive size before being detected. Therefore, cholesteatoma should be one of the working diagnosis when an elderly patient present with unilateral conductive hearing loss that is associated with tinnitus and dizziness.

\section{References}

1. Kanzara T, Virk JS, Chawda S, Owa AO. Wholly endoscopic permeatal removal of a petrous apex cholesteatoma. Case Rep Otolaryngol 2014;2014:184230.

2. Davidoss N, Ha J, Banga R, Rajan G. Delayed Presentation of a Congenital Cholesteatoma in a 64-year-old Man: Case Report and Review of the Literature. J Neurol Surg Rep 2014;75: e113-6.

3. Bennett M, Warren F, Jackson GC, Kaylie D. Congenital cholesteatoma: theories, facts, and 53 patients. Otolaryngol Clin North Am 2006;39:1081-94.

4. Sanna M, Zini C, Gamoletti R, et al. Petrous bone cholesteatoma. Skull Base Surg 1993;3:201-13.
5. Aubry K, Kovac L, Sauvaget E, et al. Our experience in the management of petrous bone cholesteatoma. Skull Base 2010;20:163-7.

6. Kong X, Wu H, Ma W, et al. Cholesteatoma in the Sellar Region Presenting as Hypopituitarism and Diabetes Insipidus. Medicine 2016;95.

7. Kumral TL, Uyar Y, Yıldırım G, et al. Does endoscopic surgery reduce recurrence of the petrous apex cholesteatoma? Indian J Otolaryngol Head Neck Surgery 2013;65:327-32.

8. Harada T, Okuno T, Mori K. A Case of Recurrent Aural Cholesteatoma Extending into the Middle Cranial Fossa. Auris Nasus Larynx 1990;16: 209-13.

9. Wayoff M. Surgical treatment of middle ear cholesteatoma. Adv Otorhinolaryngol 1987;36:1-237.

10. Triplett TM, Griffith A, Hatanpaa KJ, Barnett SL. Dermoid cyst of the infratemporal fossa: case report and review of the literature. J Neurolog Surgery Rep 2014;75:e33-7.

11. Iannella G, Savastano E, Pasquariello $B$, et al. Giant petrous bone cholesteatoma: combined microscopic surgery and an adjuvant endoscopic approach. J Neurologic Surgery Rep 2016;2:e46-9.

12. Sergi B, Galli J, Battista $M$, et al. Dealing with paediatric cholesteatoma: how we changed our management. Acta Otorhinolaryngol Ital 2014;34:138.

13. Maniu A, Harabagiu O, Schrepler MP, et al. Molecular biology of cholesteatoma. Rom J Morphol Embryol 2014;55:7-13.

14. Giannuzzi AL, Merkus P, Taibah A, Falcioni M. Congenital mastoid cholesteatoma: case series, definition, surgical key points, and literature review. Ann Otol Rhinol Laryngol 2011;120:700-6.

15. Persaud R, Hajioff D, Trinidade A, et al. Evidence-based review of aetiopathogenic theories of congenital and acquired cholesteatoma. J Laryngol Otol 2007;121:1013-9.

16. Michaels L. Biology of cholesteatoma. Otolaryngol Clin N Am 1989;22:86981.

17. Nevoux J, Lenoir M, Roger G, et al. Childhood cholesteatoma. Eur Ann Otorhinolaryngol Head Neck Dis 2010;127:143-50.

18. Mariana C, Alexandru C, Dan-Cristian G. All about Imagistic Exploration in Cholesteatoma. Maedica 2015;10:178.

19. Taranilla MTF. Magnetic Resonance Imaging (MRI) and High Resolution Computed Tomography (HRCT): Can they improve the evaluation of Middle ear cholesteatoma? 2013.

20. Vaid S, Kamble Y, Vaid N, et al. Role of magnetic resonance imaging in cholesteatoma: the Indian experience. Indian J Otolaryngol Head Neck Surg 2013;65:485-92.

21. Fitzek C, Mewes T, Fitzek S, et al. Diffusion $\square$ weighted MRI of cholesteatomas of the petrous bone. J Magnetic Res Imaging 2002;15:636-41.

22. Vercruysse JP, De Foer B, Pouillon M, et al. The value of diffusion-weighted MR imaging in the diagnosis of primary acquired and residual cholesteatoma: a surgical verified study of 100 patients. Eur Radiol 2006;16:1461-7.

23. Takahashi H. Cholesteatoma and ear surgery: an update. Kugler Publications; 2013.

24. Pyle GM, Wiet RJ. Petrous apex cholesteatoma: exteriorization vs. subtotal petrosectomy with obliteration. Skull Base Surg 1991;1:97-105.

25. Waidyasekara P, Dowthwaite SA, Stephenson E, et al. Massive Temporal Lobe Cholesteatoma. Case Rep Otolaryngol 2015;2015.

26. Burggraaff B, Luxford WM, Doyle KJ. Neurotologic treatment of acquired cholesteatoma. Otol Neurotol 1995; 16:480-5.

27. Sanna M, Pandya Y, Mancini F, et al. Petrous bone cholesteatoma: classification, management and review of the literature. Audiol Neurotol 2011;16:12436.

28. Schick B, Dlugaiczyk J. Surgery of the ear and the lateral skull base: pitfalls and complications. GMS Curr Top Otorhinolaryngol Head Neck Surg 2013; 12 .

29. Richard SA, Lan ZG, Zhang Y, You C. Accessory Nerve Schwannoma Extending to the Foramen Magnum and Mimicking Glossopharyngeal Nerve Tumor - A Case and Review of Surgical Techniques. Wrld J Neurosci 2017;7: 233.

30. Lazard DS, Tosello M, Bozorg-Grayeli A, et al. Early complications and symptoms of cerebellopontine angle tumor surgery: a prospective analysis. Eur Arch Oto-Rhino-Laryngol 2011;268: 1575-82.

31. Moffat D, Jones S, Smith W. Petrous temporal bone cholesteatoma: a new classification and long-term surgical outcomes. Skull Base 2008;18:107-15.

32. Juhn SK, Jung MK, Hoffman MD, et al. The role of inflammatory mediators in the pathogenesis of otitis media and sequelae. Clin Exp Otorhinolaryngol 2008;1:117-38. 